\title{
Inter- and intrachromosomal asynchrony of cell division cycle events in root meristem cells of Allium cepa: possible connection with gradient of cyclin B-like proteins
}

\author{
Aneta Żabka · Justyna Teresa Polit • \\ Janusz Maszewski
}

Received: 19 March 2010/Revised: 17 April 2010/Accepted: 5 May 2010/Published online: 21 May 2010

(C) The Author(s) 2010. This article is published with open access at Springerlink.com

\begin{abstract}
Alternate treatments of Allium cepa root meristems with hydroxyurea (HU) and caffeine give rise to extremely large and highly elongated cells with atypical images of mitotic divisions, including internuclear asynchrony and an unknown type of interchromosomal asynchrony observed during metaphase-to-anaphase transition. Another type of asynchrony that cannot depend solely on the increased length of cells was observed following longterm incubation of roots with HU. This kind of treatment revealed both cell nuclei entering premature mitosis and, for the first time, an uncommon form of mitotic abnormality manifested in a gradual condensation of chromatin (spanning from interphase to prometaphase). Immunocytochemical study of polykaryotic cells using anti- $\beta$ tubulin antibodies revealed severe perturbations in the microtubular organization of preprophase bands. Quantitative immunofluorescence measurements of the control cells indicate that the level of cyclin B-like proteins reaches the maximum at the G2 to metaphase transition and then becomes reduced during later stages of mitosis. After longterm incubation with low doses of $\mathrm{HU}$, the amount of cyclin B-like proteins considerably increases, and a significant number of elongated cells show gradients of these proteins spread along successive regions of the perinuclear cytoplasm. It is suggested that there may be a direct link between the effects of HU-mediated deceleration of S- and G2-phases and an enhanced concentration of cyclin B-like
\end{abstract}

Communicated by A. Feher.

\footnotetext{
A. Żabka $(\bowtie) \cdot$ J. T. Polit · J. Maszewski

Department of Cytophysiology, Institute of Physiology,

Cytology and Cytogenetics, Faculty of Biology and

Environmental Protection, University of Łódź,

Pilarskiego 14, 90-231 Łódź, Poland

e-mail: zabkka@poczta.onet.pl
}

proteins. In consequence, the activation of cyclin B-CDK complexes gives rise to an abnormal pattern of premature mitotic chromosome condensation with biphasic nuclear structures having one part of chromatin decondensed, and the other part condensed.

Keywords Allium cepa - Biphasic nuclei - Cell cycle · Chromatin - Cyclin B-like proteins · Hydroxyurea . Nuclear asynchrony $\cdot$ Preprophase band

\section{Introduction}

Although most of the cells in Eukaryotes are mononucleate, a vast number of fungi, plants, and animals are known to produce bi-, poly-, or multinucleate forms (termed "syncytia") that appear occasionally at various stages of morphogenesis (Baluška et al. 2004 and references therein). Examples of these include, but are not limited to, plasmodia in the slime mold Physarum polycephalum, endosperm in seeds of flowering plants, embryos of Drosophila melanogaster at early stages of development, and giant osteoclasts, myoblasts, or placental trophoblasts in mammals, which all create syncytia by cell fusion events. As a general rule, the emergence of a multinucleate state is correlated with synchronous mitotic divisions or mitotic waves. A number of cases relating to plants include tip cells of the thallus in coenocytic algae (e.g. Cladophora), milk tubes (laticiferous tissue) in the seedlings of Euphorbia marginata, secretory tapetum characterized by nuclear morphologies changing with respect to developmental stage, and common areas of polarized cytoplasm in cereal endosperm alveoli (Kapraun 2005 and references therein). Synchronization of mitotic divisions is also observed in groups of symplasmically interconnected 
mononucleate cells that form antheridial filaments in male sex organs of Charophytes. Plugging of plasmodesmata in the central cell wall of the filament brings about discrimination of its two physiologically separate fragments, each of them setting different rhythm of the cell cycle (Kwiatkowska and Maszewski 1986; compare also Maszewski and van Bel 1996). Consistent with the very early cell fusion experiments carried out by Rao and Johnson (1970) and more recent results (Gladfelter et al. 2006), the molecular basis of synchronous mitosis lies in the diffusion of biochemical factors (such as mitosis promoting factor, MPF, composed of a cyclin-dependent kinase and B-type cyclin; Criqui and Genschik 2002; Gotoh and Durante 2006), which spread evenly among neighboring nuclei in the common cytoplasm.

Despite a widespread relationship between the multinucleate state and intracellular coordination of nuclear processes, mitosis can also occur in a parasynchronous or asynchronous manner, which allows cell nuclei residing in the same cytoplasm to behave independently. Such type of regulation, frequently observed in filamentous fungi (e.g. Neurospora crassa and Ashbya gossypii), might be important for the proper response to nutrients and extracellular signals, more economic usage of energy and metabolites needed for replication and/or mitosis, and prevention from abrupt changes in the volumetric ratios of nuclear and cytoplasmic compartments of the cell. Accordingly, autonomous behavior of cell nuclei and their asynchronous divisions can be thought to result from spatial fluctuations in the concentration of the key cell cycle regulators (activators or inhibitors), physical asymmetries in the distribution of nuclear pore complexes, partial separation via endomembrane systems, or creation of discrete zones that promote or restrain the progression toward mitosis [reviewed by Gladfelter et al. (2006)].

In contrast to diversification of individual nuclear cycle times, generally considered in the context of an uneven concentration of macromolecular regulatory elements distributed along distant locations in the cytoplasm, most transformations confined to the interior of the nucleus are carried out in complete synchrony. Although epigenetic inheritance responsible for DNA methylation and chromatin modifications gives rise to asynchronous replication patterns of euchromatic and heterochromatic segments of chromosomes (overlapping with gene-rich and gene-poor DNA sequences, respectively), all processes required to preserve genome architecture and to promote successful progression through the cell division cycle occur simultaneously. Among many, these include licensing of DNA replication origins at the end of mitosis (Lutzmann et al. 2006), initiation events at the start of S phase (Kearsey and Labib 1998), early and late mitotic processes involved in condensation of chromosomes at prophase, and their abrupt separation and segregation during anaphase (e.g. Hirano 2000).

In plants, axially elongated cells may result from administration of chemical agents that, respectively, either strongly delay progression through DNA replication [but allow cell growth to continue (Navarrete et al. 1983; Giménez-Abián et al. 2001)] or affect formation of the phragmoplast, a highly specific cytokinetic apparatus made up of microtubules and microfilaments (Samuels and Staehelin 1996; Nishihama and Machida 2001). In order to assess the extent to which particular cell nuclei located in the same cytoplasm may display mitotic autonomy, two experimental systems effective in producing extremely large cells have been used. The first method, based on the modified procedure advanced by Giménez-Abián et al. (2001), relied on alternate incubations of primary root meristems of Allium cepa with hydroxyurea (HU, an inhibitor of ribonucleotide reductase) and caffeine ( $\mathrm{CF}$, a well-known inhibitor of cytokinesis in plants). Some of abnormally long polykaryotic cells, with two or more nuclei fused together, gave rise to atypical images of mitotic divisions, including asynchronous metaphase-toanaphase transitions. The second method, based on prolonged incubations with HU (Barlow 1969), revealed an abnormal pattern of chromosome condensation, characterized by a gradient of chromatin states extending along successive regions of a single cell nucleus from interphase to middle stages of mitosis (e.g. from G2-phase to prometaphase). Consequently, our data show for the first time that under certain conditions not only internuclear but also intranuclear and intrachromosomal course of mitotic processes may proceed asynchronously, indicating that a number of cell cycle checkpoint mechanisms must have been entirely overridden or severely constrained. In this report we also provide evidence that prolonged HU-mediated replication stress may account for an increased level of cyclin B-like proteins, and most probably, its gradient formed along the cell's axis is responsible for creating biphasic nuclear structures, having both interphase and mitotic domains of chromatin.

\section{Materials and methods}

\section{Plant material}

Seeds of Allium cepa L. (Horticulture Farm in Lubiczów) were sown on moist blotting paper and germinated at room temperature in the dark. Four days after imbibition, seedlings with primary roots ranging from 1.5 to $2 \mathrm{~cm}$ were selected and placed in Petri dishes $(\varnothing 6 \mathrm{~cm})$ filled either with $10 \mathrm{ml}$ of distilled water (control samples) or solutions applied to induce multinucleate or asynchronous cells. 
Chemical agents and treatments

All chemicals for buffering solutions, hydroxyurea (HU), bovine serum albumin (BSA), PBS, ethidium bromide, propidium iodide, pararosaniline, diazabicyclo[2.2.2]octane (DABCO), molecular weight markers, and antibodies (mouse monoclonal anti- $\beta$ tubulin antibody, goat antimouse FITC-conjugated antibody, rabbit anti-cyclin B1 IgG fraction, and goat anti-rabbit IgG FITC- and AP-conjugated antibodies) were supplied by Sigma-Aldrich (Poland). Caffeine (CF) and cellulase (Onozuka R-10) were obtained from Serva (Germany), pectinase and Triton X-100 from Fluka (Germany), and pectolyase from ICN (Costa Mesa, USA). For immunoblotting, Protease Inhibitor Cocktail (P-9599) and Coomassie blue, was purchased from SigmaAldrich (Poland), P-PER Plant Protein Extraction Kit from Pierce (Rochford, USA), Bis Tris/2-(4-morpholino)-ethanesulfonic acid SDS-NuPAGE Novex gel, polyvinylidene fluoride membrane $(0.2-\mu \mathrm{m}$ pore size), and Chromogenic Western Blot Immunodetection Kit from Invitrogen Corp. (Carlsbad, USA).

To obtain multinucleate cells, seedlings were treated according to the protocol of Giménez-Abián et al. (2001) using alternate incubations with $0.75 \mathrm{mM} \mathrm{HU}$ and $5 \mathrm{mM}$ $\mathrm{CF}$, except that additional third treatment with $\mathrm{CF}(3 \mathrm{~h})$ and post-incubation with water $(12 \mathrm{~h})$ were introduced before fixation. Induction of biphasic cells showing gradual changes of chromatin condensation (intrachromosomal asynchrony) were obtained by incubation of seedlings with $0.75 \mathrm{mM} \mathrm{HU}(12 \mathrm{~h})$, followed by continuous treatment with $0.5 \mathrm{mM} \mathrm{HU}$ (total time ranging from 24 up to $120 \mathrm{~h}$ ). During incubation with $0.5 \mathrm{mM}$ HU solutions (changed every $24 \mathrm{~h}$ ), roots were permanently aerated by gentle rotation in a water-bath shaker $\left(100 \mathrm{rpm}\right.$, at dark, $\left.23^{\circ} \mathrm{C}\right)$.

Feulgen staining and cytophotometry

Primary roots of A. cepa were fixed in cold Carnoy's mixture (absolute ethanol and glacial acetic acid; 3:1, v/v) for $1 \mathrm{~h}$, washed several times with ethanol, rehydrated, hydrolyzed in $4 \mathrm{M} \mathrm{HCl}(1 \mathrm{~h})$, and stained with Schiff's reagent (pararosaniline) according to the standard method (e.g. Polit et al. 2002). After rinsing in $\mathrm{SO}_{2}$-water (3 times) and distilled water, $1.5-\mathrm{mm}$-long apical segments were cut off, placed in a drop of $45 \%$ acetic acid and squashed onto Super-Frost (Menzel-Gläser, Braunschweig, Germany) microscope slides. Following freezing with dry ice, coverslips were removed, and the dehydrated dry slides were embedded in Canada balsam before examination. Nuclear DNA content was evaluated by means of microdensitometry using a Jenamed 2 microscope (Carl Zeiss, Jena, Germany) with the computer-aided Cytophotometer v1.2 (Forel, Lodz, Poland) for image analysis. The extinction of
Feulgen-stained cell nuclei was measured at $565 \mathrm{~nm}$ and calibrated in arbitrary units, taking the values recorded for half-telophases and prophases from control plants as reference standard of $2 \mathrm{C}$ [33.55 pg; according to Van't Hof (1965); Bennett et al. (2000)] and 4C DNA levels, respectively.

\section{Western blotting}

Root meristem cells were lysed using P-PER Plant Protein Extraction Kit supplemented with Protease Inhibitor Cocktail according to vendor's instructions. The samples were cleared by centrifugation, and total protein extracts, fractionated on 4-12\% Bis Tris/2-(4-morpholino)-ethanesulfonic acid SDS-NuPAGE Novex gel were blotted onto polyvinylidene fluoride membrane $(0.2-\mu \mathrm{m}$ pore size). Cyclin B-like proteins were detected with the rabbit polyclonal anti-cyclin B1 IgG fraction diluted to 1:300 using the Chromogenic Western Blot Immunodetection Kit.

Immunocytochemical staining of microtubules ( $\beta$ tubulin) and cyclin B-like mitotic proteins (cyclin B)

Apical parts of roots excised from the control plants, from seedlings treated using alternate incubations with $0.75 \mathrm{mM}$ $\mathrm{HU}$ and $5 \mathrm{mM} \mathrm{CF}$ and from plants exposed to prolonged HU-treatment were fixed for $45 \mathrm{~min}\left(20^{\circ} \mathrm{C}\right)$ in PBS-buffered 3.7 ( $\beta$ tubulin) or $4.0 \%$ (cyclin B) paraformaldehyde solution. Then root tips were washed three times with PBS and placed in a citric acid-buffered digestion solution $(\mathrm{pH}$ 5.0) containing $2.5 \%$ pectinase, $2.5 \%$ cellulase and $2.5 \%$ pectolyase, and incubated at $37^{\circ} \mathrm{C}$ for $15 \mathrm{~min}$. After the digestion solution was removed, root tips were washed as before, rinsed with distilled water and squashed onto Super Frost Plus glass slides (Menzel-Gläser, Braunschweig, Germany). When air-dried at room temperature, the slides were pretreated with PBS-buffered $8 \%$ BSA and $0.1 \%$ Triton X-100 for $50 \min \left(20^{\circ} \mathrm{C}\right)$ and incubated with either mouse monoclonal anti- $\beta$ tubulin antibody or rabbit anticyclin B1 IgG fraction, dissolved in PBS containing $1 \%$ BSA at a dilution of 1:750 ( $\beta$ tubulin) or 1:50 (cyclin B), respectively. Following an overnight incubation in a humidified atmosphere $\left(4^{\circ} \mathrm{C}\right)$, slides were washed three times with PBS and incubated for $1.5 \mathrm{~h}\left(18^{\circ} \mathrm{C}\right)$ with secondary goat anti-mouse FITC antibody in PBS (1:500; v/v, for $\beta$ tubulin) or goat anti-rabbit IgG [whole molecule, $\mathrm{F}\left(\mathrm{ab}^{\prime}\right)_{2}$ fragment] FITC antibody (1:500; v/v, for cyclin B). In some experimental series, cell nuclei were counterstained either with ethidium bromide $(0.4 \mu \mathrm{g} / \mathrm{ml})$ or propidium iodide $(0.3 \mu \mathrm{g} / \mathrm{ml})$. Following washing with PBS, slides were air dried and embedded in PBS:glycerol mixture (9:1) with $2.3 \%$ DABCO. Observations were made using Eclipse E-600 epifluorescence microscope (Nikon, 
Japan) equipped with B2 filter (blue light; $\lambda=465-$ $496 \mathrm{~nm}$ ) for FITC and G2 filter (green light; $\lambda=540$ / $25 \mathrm{~nm}$ ) for ethidium bromide- or propidium iodide-stained cell nuclei. All images were recorded at exactly the same time of integration using DS-Fil CCD camera (Nikon, Japan).

\section{Observations and analyses}

Feulgen-stained slides were examined, and the mitotic cells were counted using YS100 Biological Microscope (Nikon). To evaluate mean values, 4,500-5,000 cells taken from 8-10 roots were analyzed for each experimental series. Some root meristems fixed with Carnoy's mixture were hydrolyzed with $4 \mathrm{M} \mathrm{HCl}(1 \mathrm{~h})$, placed in a drop of $45 \%$ acetic acid, squashed onto microscope slides, and unstained cells were observed using Optiphot-2 microscope (Nikon) equipped with phase contrast optics and DXM 1200 CCD camera (Nikon, Japan). Quantitative measurements of cyclin B-like protein immunofluorescence were made after converting color images into gray scale and expressed in arbitrary units as mean pixel value (pv) spanning the range from 0 (dark) to 255 (white).

All experiments performed using immunofluorescence methods were repeated at least twice, others several times, and the most representative results were selected for reporting here.

\section{Results and discussion}

Repeated incubations of primary root meristems of Allium cepa with caffeine (to inhibit cell plate formation) and then with hydroxyurea (to stop DNA replication and to increase the length of the cell) have been shown effective in producing polykaryotic cells that advance the metaphaseanaphase breakpoint synchronously, despite previous differences in chromosome condensation (Giménez-Abián et al. 2001). Our results indicate that using a modified version of this method (with extended periods of HU and CF treatment) two dominant types of cells can be formed: one, comprising various numbers of nuclei $(1,2,4,6$, and sporadically 8; each nucleus containing up to 4C DNA content; Fig. 1a), and the other, mononuclear cells, with increased C-values or chromosome numbers (Fig. 1b, c). Quantitative analysis of the first cell type, performed $72 \mathrm{~h}$ after the start of alternating HU-CF incubations, demonstrates a correlation between the genome copy number of the cell (or total ploidy level) and the incidence of nuclear asynchrony (Fig. 2). The higher the number of nuclei (and the longer the cell), the greater the frequency of evident differences between the various states of chromosome condensation or between the discrete stages at which
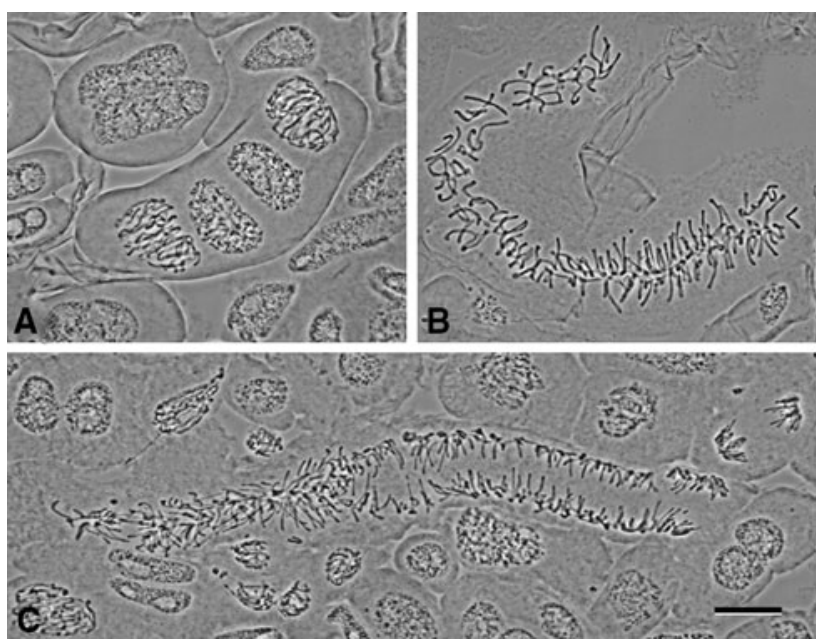

Fig. 1 Elongated root meristem cells of Allium cepa following 50-h alternate incubations with hydroxyurea and caffeine; phase-contrast microscopy. a Internuclear asynchrony (various stages of prophase) showing acceleration of mitotic chromosome condensation in polar nuclei surrounded by relatively large amounts of the cytoplasm; interchromosomal asynchrony during $\mathbf{b}$ prometaphase-to-metaphase and $\mathbf{c}$ metaphase-to-anaphase transition. Bar $20 \mu \mathrm{m}$

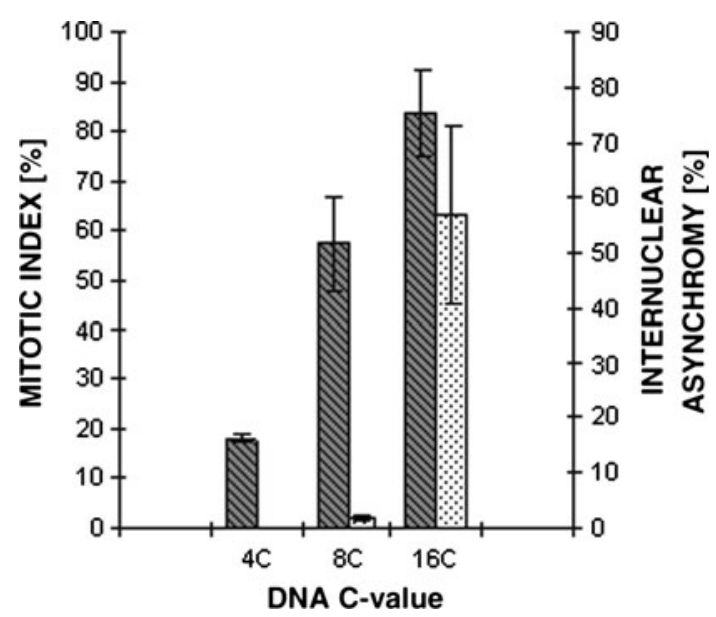

Fig. 2 Mitotic indices (\%) (diagonal lined bars) and relative numbers of M-phase cells showing internuclear asynchrony (\%) (dotted diagrams) in root meristem cells of A. cepa having various total DNA C-values (4C, 8C, and 16C; cytophotometric evaluations of Feulgen-stained cell nuclei) after 50-h alternate incubations with hydroxyurea and caffeine. Each mean value based on the analysis of ten root meristems $(>2,000$ cells/meristem)

individual nuclei can be observed passing through mitosis. According to the data presented by Giménez-Abián et al. (2001), the advancement of chromosome condensation has been often noticed within the outlying parts of giant multinucleate cells (in more than $90 \%$ of cases; Fig. 1a), indicating that acceleration of mitosis is due mainly to relatively high amount of surrounding cytoplasm, compared with the perinuclear cytoplasm located in middle part of the cell. In the second type of cells, derived from about 
Fig. 3 Immunocytochemical localization of $\beta$ tubulin in root meristem cells of $A$. cepa. a Late G2 phase control cell showing equatorial position of PPB (left) and anaphase cell demonstrating microtubular arrays of spindle fibers extending between two polar areas (right). Bar $10 \mu \mathrm{m}$.

b, c Irregular and sinusoidal microtubular ribbons of PPBs in polykaryotic cells. Bar $20 \mu \mathrm{m}$. d, e Elongated cells showing interchromosomal asynchrony with discrete regions varying in the intensity of $\beta$ tubulin immunofluorescence during prometaphase (d) and anaphase (e); arrows depict local concentrations of microtubules. Bar $20 \mu \mathrm{m}$. f Prolonged treatment with $\mathrm{HU}$; microtubular arrays in anaphase cell with evident symptoms of premature chromosome condensation (left) and asymmetric localization of PPB in a cell showing gradual condensation of chromatin (right). Bar $20 \mu \mathrm{m}$
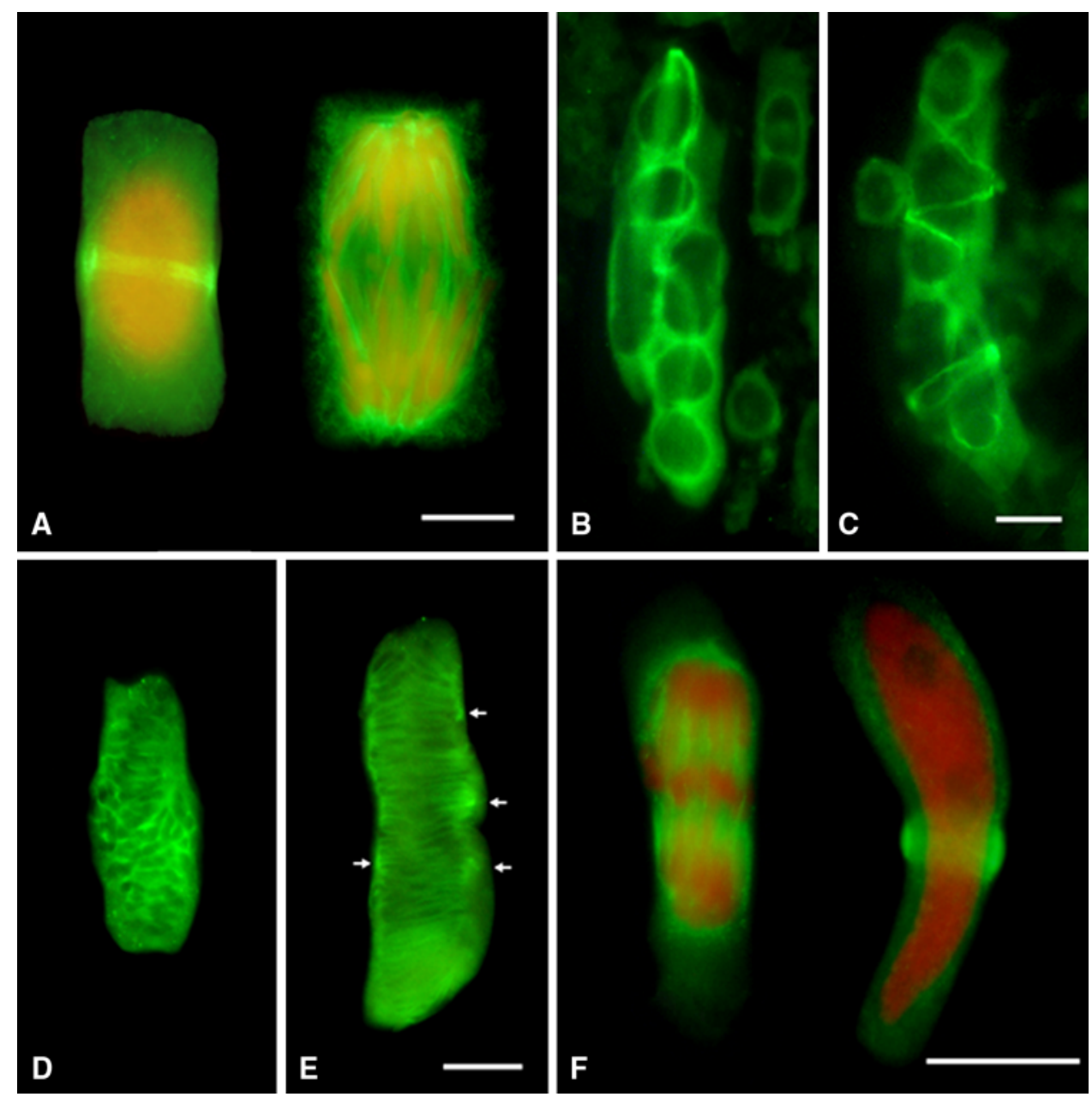

$15 \%$ of all polykaryons, the nuclei compressed to the center of the cell become fused together. A series of observations have demonstrated that some of the resultant cells may enter nuclear divisions asynchronously, displaying a stepwise progression of successive phases of mitosis. In such cases, transitions from prometaphase to metaphase (Fig. 1b), or from metaphase to anaphase (Fig. 1c), seemed to proceed either gradually, or with only slightly discernible intermediate phase-to-phase transition zone between two adjoined tetraploid groups of chromosomes.

The changing arrangements of microtubular arrays establish highly specific landmarks for the different stages of mitosis (Dhonukshe and Gadella 2003). Our immunocytochemical studies using anti- $\beta$ tubulin antibodies (Fig. 3) revealed severe perturbations of preprophase bands (PPBs) formed in polykaryotic cells generated by the recurring HU-CF treatments. As compared with the control late-G2 cells, which displayed typical ring-shaped microtubular arrays (Fig. 3a), most of multinuceate cells demonstrated either irregular bands of microtubules stretching beneath the long cell wall (Fig. 3b), or sinusoidal microtubular ribbons penetrating cortical layers of the cytoplasm (Fig. 3c). During prometaphase and metaphase (Fig. 3d), elongated cells with more or less evident interchromosomal asynchrony displayed few regions varying in the intensity of $\beta$ tubulin immunofluorescence. At later stages (during anaphase), diffuse spindles have appeared, a number of them containing several distinct areas of microtubular concentrations (Fig. 3e, arrows; compare with the control anaphase cell in Fig. 3a).

Long-term incubation with low doses of hydroxyurea (ranging from 0.75 to $0.50 \mathrm{mM}$ ) has proved to be another type of treatment effective in producing large and elongated cells in primary root meristems of $A$. cepa (compare also Barlow 1969). Surprisingly, this kind of influence has revealed the most significant changes and severe perturbations to the process of mitotic chromatin condensation that cannot be accounted for solely by the increased amount of cytoplasm in the polar regions of the cell. In spite of the fact that root cells treated up to $72 \mathrm{~h}$ with $0.75 /$ $0.50 \mathrm{mM}$ HU continuously accumulated in late S- and G2phases (Fig. 4a, b), within the whole period of treatment a relatively small amount of mitotic divisions has been 

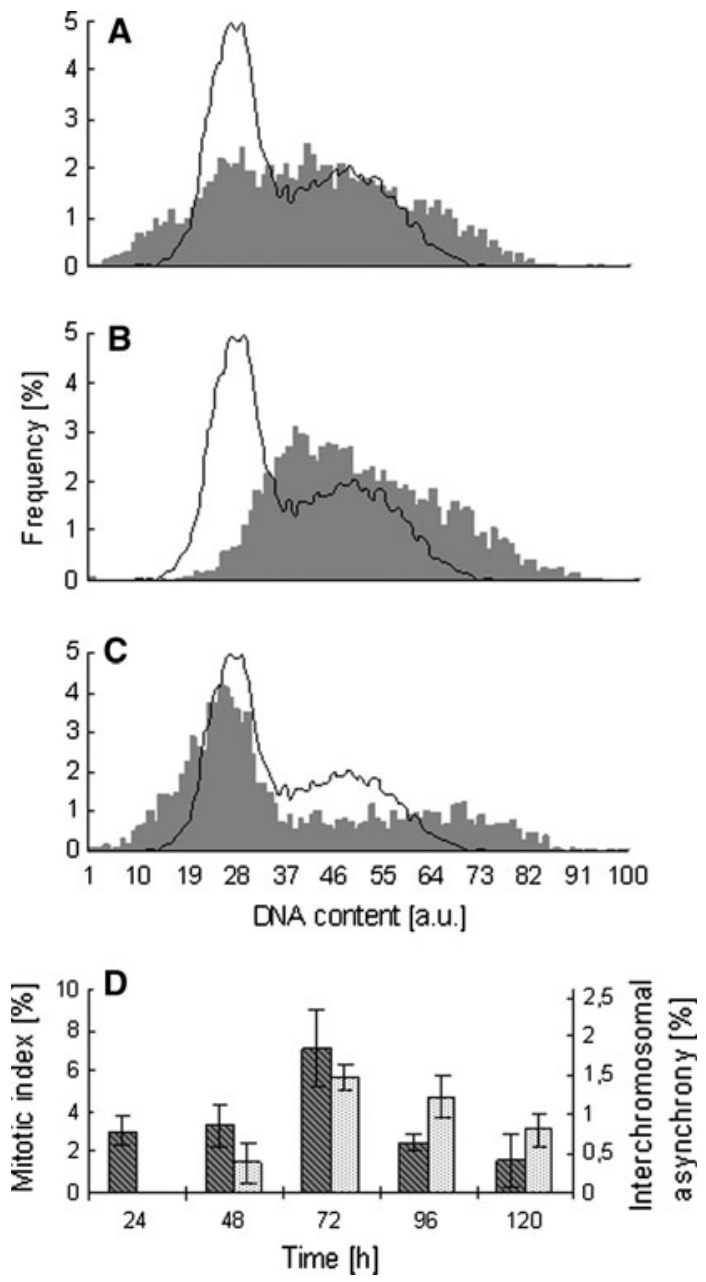

Fig. 4 The effect of prolonged incubation with $0.75 / 0.50 \mathrm{mM}$ HU on cell populations in root meristems of $A$. серa. a-c Frequency distributions (\%) for nuclear DNA contents (Feulgen-staining; arbitrary units, a.u.) following $24 \mathrm{~h} \mathrm{(a),} 72 \mathrm{~h}$ (b), and $120 \mathrm{~h}$ (c) treatment (gray diagrams), in comparison with the control (lined graph at each diagram shows the frequency of G1, S, and G2 cells within a population of untreated seedlings). d Mitotic indices [(\%), lined diagrams] and relative numbers of M-phase cells showing intrachromosomal asynchrony [(\%), dotted diagrams; total duration of treatment (Time, h)]. Each mean value calculated from ten root meristems $(>2,000$ cells/meristem)

constantly observed (with a significant increase $72 \mathrm{~h}$ after the start of incubation; Fig. 4d), most of them indicating apparent symptoms of premature chromosome condensation (PCC; Fig. 5). Induction of PCC, easily recognized in meta-, ana- and telophase (Fig. 5d-h), was restricted merely to a relatively small subpopulation of cells having their DNA almost completely replicated (Fig. 4b). Accordingly, while a considerable drop in the frequency of M-phase cells reflects the secondary effect of the block imposed upon DNA synthesis, then on the contrary, a minor fraction of cells entering PCC has to be viewed as a consequence of mechanisms which permit these cells to
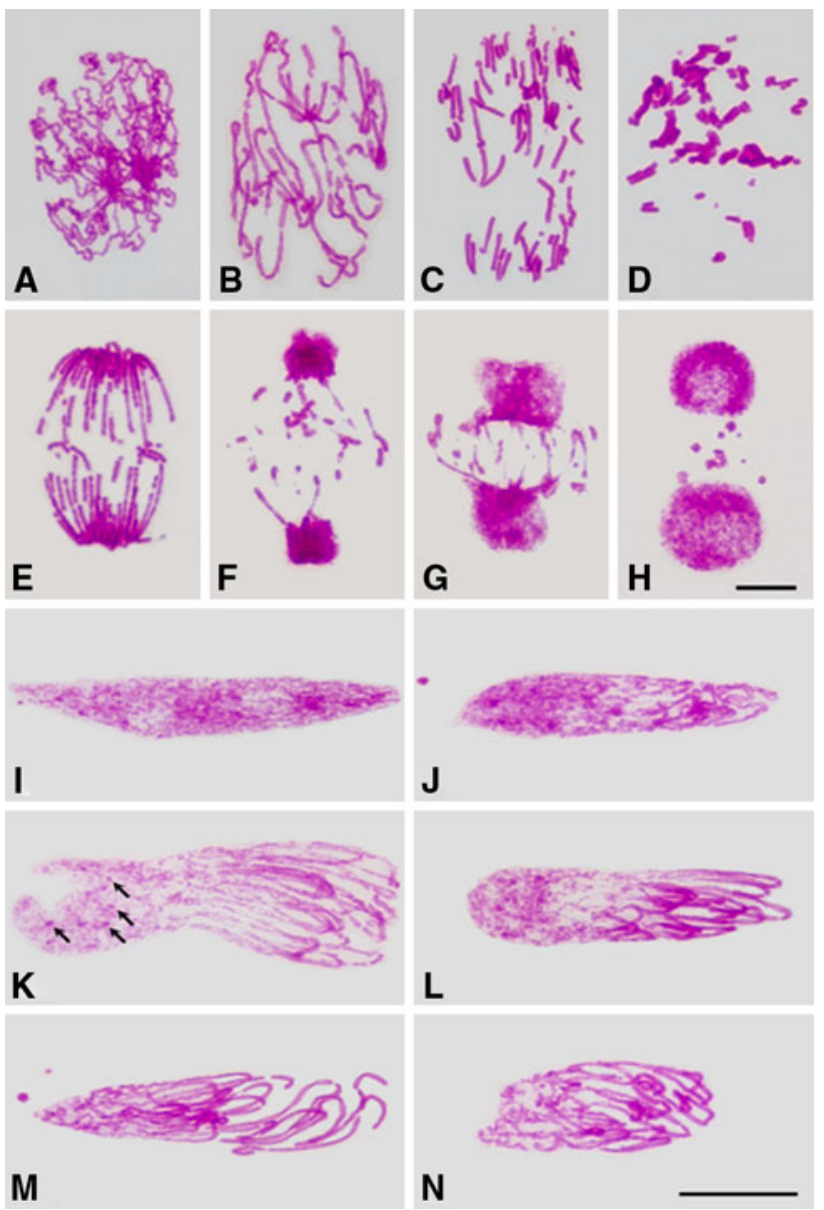

Fig. 5 Nuclear Feulgen-staining of A. cepa root meristem cells following 72-h treatment with 0.75/0.50 mM HU. Bar $10 \mu \mathrm{m}$. a-h Cell nuclei with evident symptoms of premature chromosome condensation (PCC) at early (a) and late prophase (b), prometaphase (c), metaphase (d), anaphase (e), early (f) and late telophase (g), and post-telophase (h). $\mathbf{i}-\mathbf{n}$ Intrachromosomal asynchrony in cells showing evident gradients of chromatin condensation: progressive condensation of chromatin observed during transitions from interphase to early prophase $(\mathbf{i}, \mathbf{j})$, from interphase-to-prophase/prometaphase $(\mathbf{k}, \mathbf{l})$, and from early prophase to prometaphase $(\mathbf{m}, \mathbf{n})$. Darkly stained chromocenters corresponding to telomeric heterochromatin indicated by arrows $(\mathbf{k})$

override the DNA stress-response pathway (also referred to as the S-phase checkpoint; Osborn et al. 2002) and allow them to proceed toward premature mitosis regardless of the incomplete replication of nuclear DNA (Kohn et al. 2002; Rybaczek and Maszewski 2007a, b).

Together with cells showing typical features of an unscheduled mitotic division, such as chromosomal gaps and breaks, lagging chromatids, acentric fragments of chromosomes, and micronuclei (Fig. 5a-h), about $19 \%$ of PCC cells revealed an abnormal pattern of chromosome condensation, characterized by a gradient of chromatin states along successive regions of the nucleus (Fig. 5i-n). The total DNA content measured by quantitative 
microdensitometry ranged from 30.4 to $34.9 \mathrm{pg}$, indicating almost complete replication of the diploid genome. Evident alterations in chromatin architecture could be observed from interphase to early stages of prophase (Fig. 5i, j), and from early to late stages of prophase (Fig. 5m, n). Some of the cells, however, displayed much larger array of chromatin morphologies, spanning from a highly decondensed form in interphase up to strong condensation of individual chromosomes, comparable to that observed during late prophase and prometaphase in the control roots (Fig. 5k, 1). Although cells with evident symptoms of PCC revealed mitotic spindles with regular arrays of microtubules (Fig. 3f), the most common site of PPBs in cells showing progressive gradients of chromatin condensation correlated well with a discrete area of chromatin positioned close to the transitory region between the interphase states of decondensed nucleoplasm and the chromatin structures characteristic for the initial stages of mitotic compaction at early prophase (Fig. 3f). In consequence, some PPBs have become translocated from the equatorial plane of the cell into new asymmetric positions, demarcating two unequal parts of the nucleus.

The mechanisms that define the positioning and changing dynamics of microtubular arrays (PPB, spindle, phragmoplast, and cortical microtubules) remain still unknown. According to a number of approaches adopted to demonstrate links between the regulatory factors of the cell cycle (such as MPF) and the specific microtubular systems in plants (Colasanti et al. 1993; Mineyuki et al. 1996; Hemsley et al. 2001; Van Damme 2009), it might be assumed that the exact location of PPB in HU-treated cells of Allium corresponds with a definite spatio-temporal threshold concentration of the cyclin-dependent protein kinases (CDKs). On the contrary, however, another set of experiments, by applying microinjection of $\operatorname{cdc} 2$, points to a destructive rather than constructive function of CDKs on PPB formation (Hush et al. 1996).

Different types of cyclins are the cell cycle stage-specific activators of Cdks, and their mutual interactions have been long recognized as indispensable for the ability of cells to progress from one phase to the next (Criqui et al. 2001; Francis 2007; Boruc et al. 2010). Apart from a common N-terminal motif called the "cyclin box" (a sequence of 100 amino acids), mitotic cyclins contain the "destruction box" susceptible to ubiquitination and essential for the rapid turnover of the protein (King et al. 1996). To find out possible relationship between the various types of nuclear asynchrony and an uneven intracellular distribution of one of the most critical factors engaged in the G2-to-M transition, we have detected cyclin B-like mitotic proteins by using rabbit polyclonal anti-cyclin B1 antibody (Figs. 6, 7); its specificity was demonstrated using Western blot analysis of root meristem lysates (Fig. 8). As it was shown previously, plant cyclins share a great deal of homology with the amino acid sequences of the corresponding animal proteins (particularly in the conserved consensus motifs of the cyclin box; Renaudin et al. 1994), and reveal both common epitopes and evident functional similarity between the widely divergent species of Eukaryotes (Chaudhuri and Ghosh 1997; Sen and Ghosh 1998). In our immunoblotting experiments with samples from both control and HU-treated root meristem cell lysates, anti-cyclin B1 antibody detected only one strong band at the position equivalent to a molecular weight of $\sim 54 \mathrm{kDa}$ (compare with Chaudhuri and Ghosh 1997). Long polykaryotic cells observed in onion root meristems after the alternate $\mathrm{HU} / \mathrm{CF}$ treatments displayed predominantly cytoplasmic localization of cyclin B-like proteins, concentrated mainly at the peripheries of interphase nuclei. Similar ring-like localization of RFP- and GFP-tagged cyclin B1;2, corresponding to either the nuclear envelope during interphase or the prophase spindle, has been reported by Boruc et al. (2010) in both tobacco (BY2 cell line) and Arabidopsis. The ring-shaped immunofluorescence increased considerably around cell nuclei in late G2 and early prophase, in comparison with the "delayed" nuclei located in the middle part of the cell (Fig. 6). Furthermore, the advancement of chromosome condensation in nuclei placed within the outlying parts of giant cells has been often correlated with at least partial translocation of cyclin B-like proteins into the vicinity of interchromatin domains of the nucleoplasm (Fig. 6a, a", b, b"). However, in contrast to CYCB1;2-GFP localizations observed in Arabidopsis (Boruc et al. 2010), or cyclin B1 association with HeLa S3 TK ${ }^{-}$human cells (Pines and Hunter 1991), no evident immunofluorescence signals of cyclin B-like proteins could be found in metaphase chromosomes of Allium cepa, probably due to low capacity of FITC-labeled antibody to penetrate the highly condensed structures of mitotic chromatin.

Another type of immunolabeling, with respect to both the intensity of fluorescence and localization of cyclin B-like proteins, has been observed in root meristem cells of A. cepa following long-term incubation with $0.75 / 0.50 \mathrm{mM}$ HU (Fig. 7). Direct microscopic examination (Fig. 7a, b) and quantitative data derived from the total immunofluorescence measurements in the control cells (Fig. 9) clearly depict that the level of cyclin B-like proteins reaches the maximum at the transition from late $\mathrm{G} 2$ to metaphase and then becomes reduced during later stages of mitosis. In comparison to the untreated cells, the amount of immunofluorescence increased more than 65 and 63\%, respectively, among the $\mathrm{G} 1$ and $\mathrm{G} 2$ cells in roots incubated with low doses of hydroxyurea (Figs. 7c, 9). While the majority of HU-treated cells (after third incubation) revealed typical, ring-shaped distribution of immunofluorescence, a vast 
Fig. 6 Localization of cyclin B-like proteins in long polykaryotic root meristem cells of A. cepa observed following 50-h alternate $\mathrm{HU} / \mathrm{CF}$ treatments $(\mathbf{a}, \mathbf{b})$. Note an increased ring-shaped immunofluorescence around late G2 (a) and prophase (b) nuclei located at the polar regions of the cytoplasm. Propidium iodide-stained nuclear DNA and merged images of the same cells are shown in $\mathbf{a}^{\prime}, \mathbf{b}^{\prime}$ and $\mathbf{a}^{\prime \prime}, \mathbf{b}^{\prime \prime}$, respectively. Bar $20 \mu \mathrm{m}$
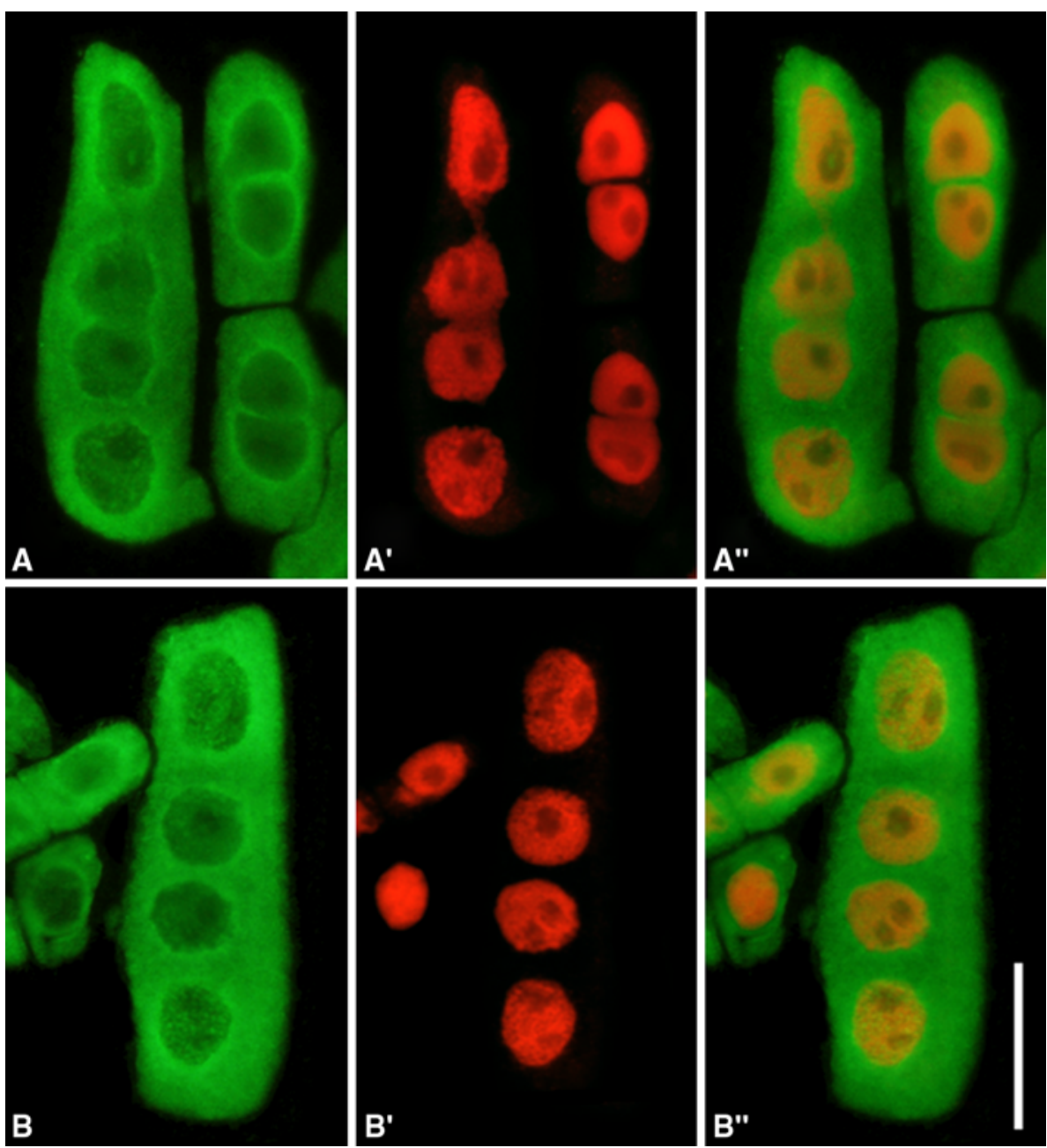

number of large, elongated cells (some of them with an abnormal pattern of chromosome condensation) displayed evident gradients of cyclin B-like proteins spread along successive regions of the nucleus (Fig. 7d, e). An example given in Fig. 7d, which shows quantitative measurements of fluorescence scanned throughout successive lines perpendicular to the late-G2 cell's axis (horizontal line), points out the nearly 50 and $33 \%$ gradual increase in mean intensity of immunolabeling of the nucleoplasm (shorter dotted line) and cytoplasm (longer dotted line), respectively, at the distance of about $50 \mu \mathrm{m}$ from both ends of the nucleus. Although most of such cases have been observed prior to the onset of mitosis (Fig. 7d), an uneven density of immunofluorescent labeling has also been observed around cell nuclei showing abnormal profile of chromatin condensation, combining attributes of interphase and prophase or displaying transitions from early to later stages of mitotic condensation (Fig. 7e).

Based on the obtained results and a number of functional parallels concerning molecular factors by which various types of cells acquire the ability to progress throughout successive stages of mitotic division (including cell cyclespecific transcription, activation, localization, and degradation of these factors), cyclin B-like proteins seem to be a good candidate to account for the abnormal course of chromosome condensation observed in seedlings of $A$. cepa. Although there is considerable controversy regarding the effects of HU on the intracellular level of cyclins (e.g. Florensa et al. 2003; Rodríguez-Bravo et al. 2007), an increased immunofluorescence observed in onion root tip cells (both in interphase and mitosis) fits well with the data showing a pronounced accumulation of all or most types of cyclins, including type B-cyclin, in response to inhibitors of DNA replication (e.g. in SK-N-MC neuroepithelioma (Fung et al. 2002), HeLa, and Chinese hamster ovary (CHO) cells (Kung et al. 1993; Gao and Richardson 2001). While it is far too early to formulate any simplified hypothesis concerning the role of specific cyclin and of its specific distribution pattern in creating mononuclear heterophasic cells, we also seem to have some theoretical grounds that allow us to link the effects of HU-mediated deceleration of S- and G2-phases with an enhanced 

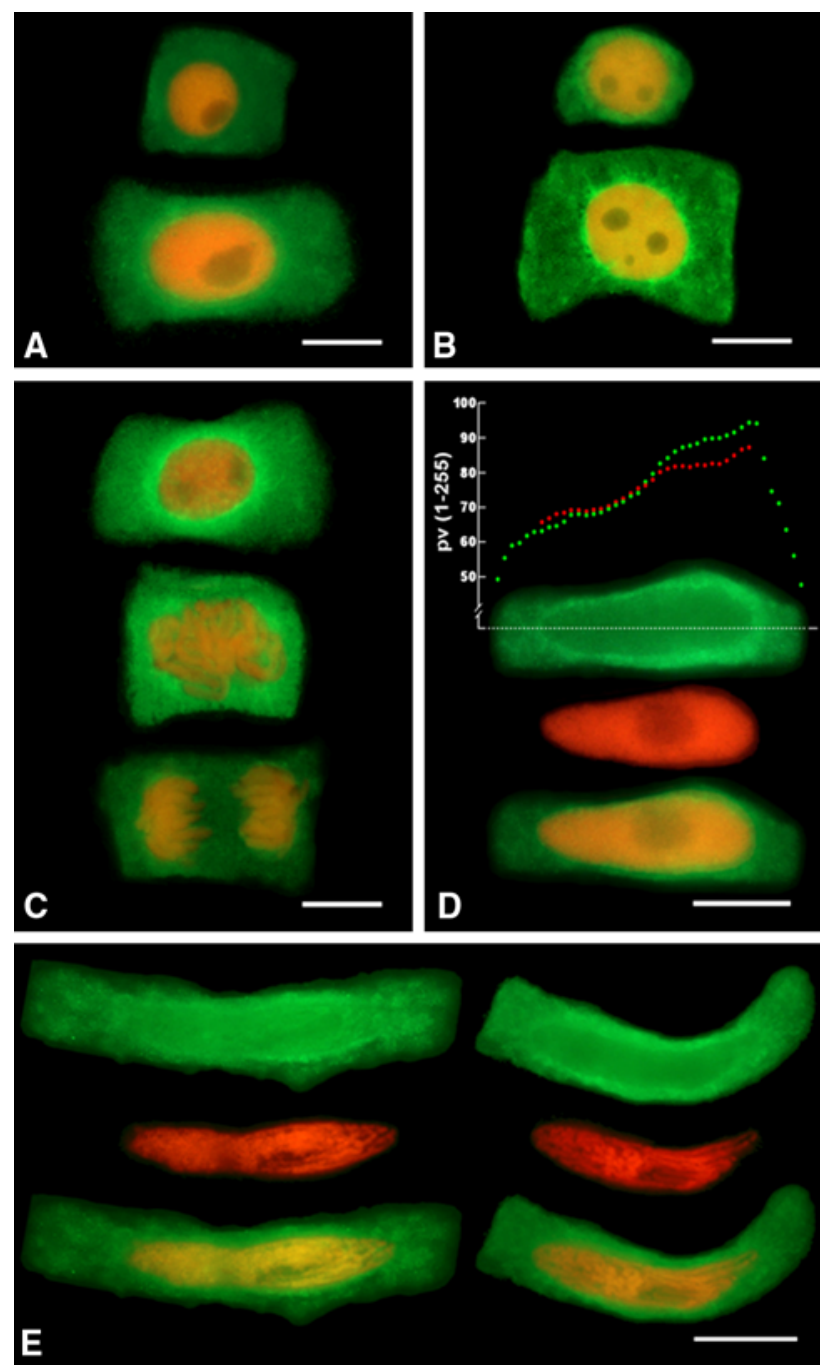

Fig. 7 Immunocytochemical localization of cyclin B-like proteins in root meristem cells of A. cepa. a, b Control interphase cells; $\mathbf{c}-\mathbf{e}$ cells after $72 \mathrm{~h}$ treatment with $0.75 / 0.50 \mathrm{mM} \mathrm{HU}$; a G1- (upper) and G2phase (lower) cells, b mitotic cells in early prophase (upper), nearmetaphase (middle), and anaphase (lower). Bar $10 \mu \mathrm{m}$. c Increased level of cyclin B-like proteins in G1- (upper) and G2-phase (lower) cells. Bar $10 \mu \mathrm{m}$. d Elongated late-G2 cell showing gradual increase of cyclin B-like proteins along the nucleus; an overlaid plot obtained by quantitative immunofluorescence scanning throughout successive lines perpendicular to the cell's axis (horizontal line). Mean intensities of nucleoplasm (shorter dotted line) and cytoplasm (longer dotted line) immunolabeling were evaluated as pixel values (pv) ranging from 0 (dark) to 255 (white), at a distance of about $50 \mu \mathrm{m}$ spanning both ends of the nucleus. Propidium iodide staining and merged images are shown in the middle and lower photographs, respectively. Bar $20 \mu \mathrm{m}$. e Uneven distribution of cyclin B-like proteins along biphasic nuclear structures, having both late $\mathrm{G} 2$ and mitotic domains of chromatin (left micrographs arranged vertically: immunofluorescence, propidium iodide, and merged image) and early/late prophase chromosomes (corresponding right micrographs). Bar $20 \mu \mathrm{m}$

concentration of cyclin B-like proteins and, consequently, with the activation of cyclin B-CDK complexes and the premature induction of mitotic chromosome condensation.
Fig. 8 Immumoblotting analysis of cyclin B-like proteins in lysates of Allium cepa root meristem cells. Lane A molecular weight marker, lane $B$ SDSNuPAGE electrophoresis and Coomasie staining, lane $C$ Western blot of separated and electrotransferred proteins performed using primary rabbit anti-cyclin B1 IgG fraction and secondary goat anti-rabbit IgG antibody conjugated with alkaline phosphatase
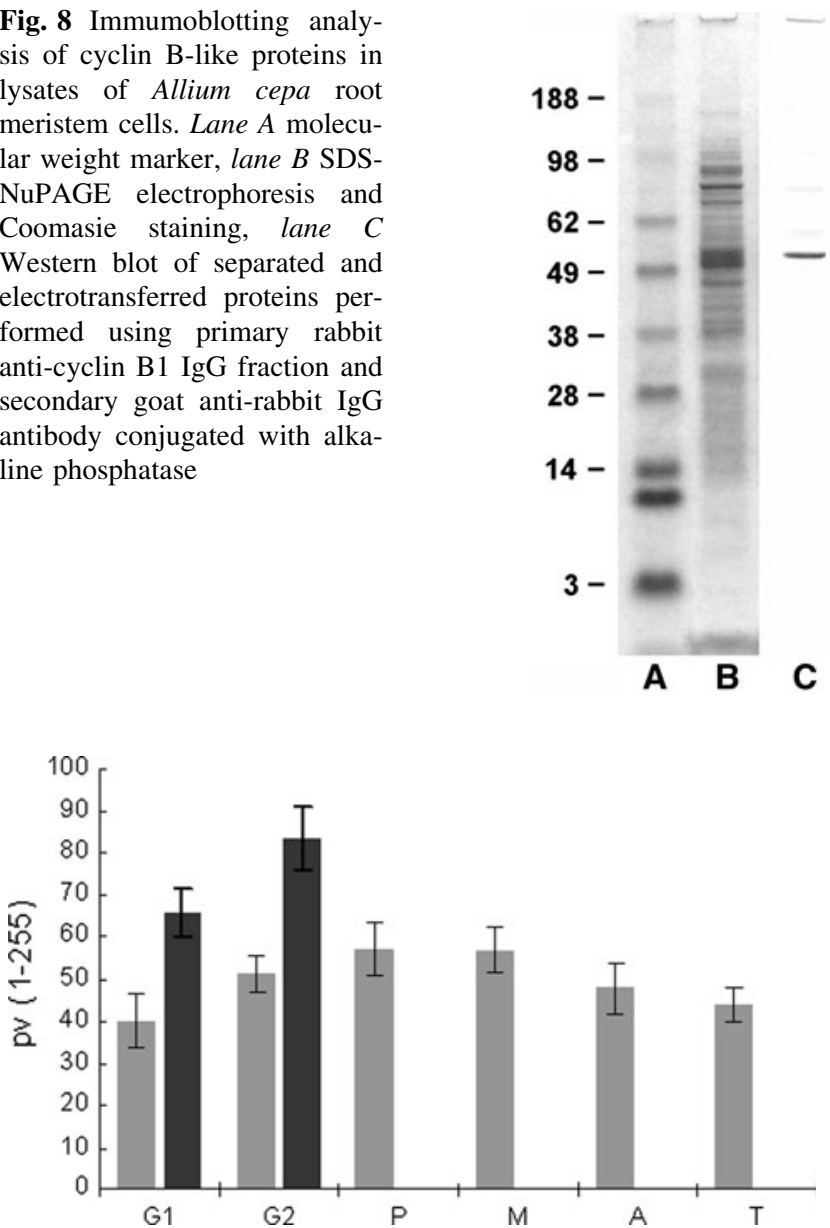

Fig. 9 Quantitative measurements of total cyclin B-like protein immunofluorescence in root meristem cells of A. cepa, expressed as mean pixel value (pv) spanning the range from 0 (dark) to 255 (white); control cells (gray diagrams) in G1-, G2-phase, prophase $(P)$, metaphase $(M)$, anaphase $(A)$, and telophase $(T)$, and G1-, G2-phase cells after the 72-h treatment with $0.75 / 0.50 \mathrm{mM} \mathrm{HU}$ (black diagrams). Each mean value based on the analysis of five root meristems ( $>100$ cells/meristem)

A vast number of secondary effects associated with HU-mediated inhibition of ribonucleotide reductase (which plays an essential role in the control of DNA replication and repair systems; Chabes and Thelander 2000) and the resultant block imposed upon replication forks involve accumulation of hemireplicated intermediates with long single-stranded ssDNA, DNA double strand breaks (DSBs) (Merrill and Holm 1999; Rybaczek and Maszewski 2007a, b), Holliday junctions (Sogo et al. 2002), and other specific DNA damage by forming nitric oxide and hydrogen peroxide (Sakano et al. 2001). To respond to these potentially life-threatening insults, cells have evolved a DNA-replication stress-response pathway, also referred to as the DNA-replication checkpoint or S-phase checkpoint. It may be assumed, however, that low concentration of $\mathrm{HU}$ (applied in our experiments) slows down cell cycle 
progression by affecting both replication forks and postreplication DNA repair processes, without any significant effect on transcription, protein synthesis, and cell growth. So, even if there were DNA replication checkpoint or S-M checkpoint mechanisms set in motion by the S-phase control system, the prolonged time of continuing accumulation of cyclin B-like proteins can give the cells enough time to reach the threshold dose of active cyclin B-type/ CDK-B complexes needed for mitotic stimulation.

The various types of asynchrony observed in root meristem cells of Allium cepa can be broadly divided into the following three categories: (a) internuclear asynchrony (found in large polykaryotic cells), (b) interchromosomal asynchrony manifested by an uneven progression of chromosomes throughout successive mitotic stages (resolved after nuclear fusion events), and (c) intrachromosomal asynchrony (creating a gradient of increasing chromatin condensation after prolonged treatment with $\mathrm{HU}$ ). All these events can be clearly linked to an extended period of experimental treatment and, accordingly, may be seen as a response to a diversified set of intracellular conditions which arise in and spread along the cytoplasm of extremely long cells. It is immediately obvious, however, that some other variable conditions are needed to convincingly explain an intrachromosomal asynchrony induced in root meristem cells after the prolonged incubation with hydroxyurea. Irrespective of the fact that no satisfactory solution may be proposed to elucidate such an unusual mode of mitotic chromosome condensation, any causal inference (although based on a speculative evaluation) must inevitably meet the challenge of two problems: (1) why and how do the cells escape safeguard mechanisms designed to block progression toward mitosis under conditions that generate stalled replication forks and DNA breakage, and (2) how do the cells rearrange their intrinsic pattern of successive cell cycle events to create, at one time, an unusual spatio-temporal continuum of nuclear modifications. To resolve the first problem, there is a need to accept functional predominance of mitotic activators (such as A- and B-type CDKs bound to A, B, and D cyclins; Francis 2007) over those factors (such as ATM and ATR kinases; Rybaczek and Maszewski 2007a, b), which efficiently promote replication checkpoint control mechanisms to prevent cells from entering nuclear division.

Perhaps, there is another morphological aspect that is worth relating in the context of the second question. The majority of mitotic chromosomes emerging in cells with biphasic chromatin structure (having one part of it decondensed, and the other part condensed) display predominantly the loop-like morphology, at the same time as the remaining half of the nucleus exhibits darkly stained chromocenters (Fig. 5k). Such appearance may indicate that chromosomes of the HU-treated root meristem cells start to condense in the vicinity of pericentromeric (proximal) regions, with their distal parts comprising telomeres retarded at earlier stages of the cell cycle. Notably, chromatin within the interphase nucleus of Allium cepa is polarized, and thus, effectively isolating centromeres from the distal telomeric regions, which include major C-bands of late-replicating heterochromatin (Fussell 1975; Fujishige and Taniguchi 1998). Moreover, most recent experiments indicate that the mitotic portions of biphasic nuclei reveal characteristic pericentromeric immunofluorescence of phosphorylated H3 histones (Ser10) (Żabka, unpublished).

Progressing upwards through the structural hierarchy of condensing chromatin along the chromosomal axis creates a host of new questions about relations between form and function rather than provides useful means to make explanations. Whatever the mechanism might prove to be, a wide range of structural changes extending from interphase to mitosis implicate continuing changes in nucleosomal and higher-order chromatin folding at the route toward the G2/M transition [combined with underlying gradients of post-translational modifications of the core and linker histones; reviewed by Jasencakova et al. (2001)], gradual transition from nuclear matrix to chromosomal scaffold, and creation of discrete zones where preprophase band is formed at late G2 and nuclear envelope breaks down in early prophase (John 1996 and references therein). Even so, however, the above two types of asynchrony may also depend on some plant-specific regulatory systems of cell cycle progression, including specific mechanisms of plant histogenesis and phytohormonal signaling, which operating in the context of root growth and development, may form dissimilar conditions for activation of the key stimulators of mitosis.

Acknowledgments This work was supported by a grant from Ministry of Science and Higher Education (N N303 503038).

Open Access This article is distributed under the terms of the Creative Commons Attribution Noncommercial License which permits any noncommercial use, distribution, and reproduction in any medium, provided the original author(s) and source are credited.

\section{References}

Baluška F, Volkmann D, Barlow PW (2004) Eukaryotic cells and their cell bodies: cell theory revised. Ann Bot 94:9-32

Barlow PW (1969) Cell growth in the absence of division in a root meristem. Planta 88:215-223

Bennett MD, Johnston S, Hodnett GL, Price HJ (2000) Allium cepa L. cultivars from four continents compared by flow cytometry show nuclear DNA constancy. Ann Bot 85:351-357

Boruc J, Mylle E, Duda M, De Clercq R, Rombauts S, Geelen D, Hilson P, Dirk I, Van Damme D, Russinova E (2010) Systematic localization of the Arabidopsis core cell cycle proteins reveals novel cell division complexes. Plant Physiol 152:553-565 
Chabes A, Thelander L (2000) Controlled protein degradation regulates ribonucleotide reductase activity in proliferating mammalian cells during the normal cell cycle and in response to DNA damage and replication blocks. J Biol Chem 275:1774717753

Chaudhuri SK, Ghosh S (1997) Monoclonal antibody raised against human mitotic cyclin B1. identifies cyclin B-like mitotic proteins in synchronized onion (Allium cepa L.) root meristem. Cell Biol Int 21:159-166

Colasanti J, Cho S-O, Wick S, Sundaresan V (1993) Localization of the functional $\mathrm{p} 34^{\mathrm{cdc} 2}$ homolog of maize in root tip and stomatal complex cells: association with predicted division sites. Plant Cell 5:1101-1111

Criqui MC, Genschik P (2002) Mitosis in plants: how far we have come at the molecular level? Curr Opin Plant Biol 5:487-493

Criqui MC, Weingartner M, Capron A, Parmentier Y, Shen W-H, Heberle-Bors E, Bögre L, Genschik P (2001) Sub-cellular localisation of GFP-tagged tobacco mitotic cyclins during the cell cycle and after spindle checkpoint activation. Plant $\mathrm{J}$ 28:569-581

Dhonukshe P, Gadella TWJ Jr (2003) Alteration of microtubule dynamic instability during preprophase band formation revealed by yellow fluorescent protein-CLIP170 microtubule plus-end labeling. Plant Cell 15:597-611

Florensa R, Bachs O, Agell N (2003) ATM/ATR-independent inhibition of cyclin B accumulation in response to hydroxyurea in nontransformed cell lines is altered in tumour cell lines. Oncogene 22:8283-8292

Francis D (2007) The plant cell cycle-15 years on. New Phytol 174:261-278

Fujishige I, Taniguchi K (1998) Sequence of DNA replication in Allium fistulosum chromosomes during S-phase. Chromosome Res 6:611-619

Fung TK, Siu WY, Yam CH, Lau A, Poon RYC (2002) Cyclin F is degraded during G2-M by mechanisms fundamentally different from other cyclins. J Biol Chem 277:35140-35149

Fussell CP (1975) The position of interphase chromosomes and late replicating DNA in centromere and telomere regions of Allium cepa L. Chromosoma 50:201-210

Gao J, Richardson DR (2001) The potential of iron chelators of the pyridoxal isonicotinoyl hydrazone class as effective antiproliferative agents, IV: the mechanisms involved in inhibiting cellcycle progression. Blood 98:842-850

Giménez-Abián JF, Clarce DJ, Giménez-Abián MI, de la Torre C, Giménez-Martin G (2001) Synchronous nuclear-envelope breakdown and anaphase onset in plant multinucleate cells. Protoplasma 218:192-202

Gladfelter AS, Hungerbuehler AK, Philippsen P (2006) Asynchronous nuclear division cycles in multinucleated cells. J Cell Biol 172:347-362

Gotoh E, Durante M (2006) Chromosome condensation outside of mitosis: mechanisms and new tools. J Cell Physiol 209:297-304

Hemsley R, McCutcheon S, Doonan J, Lloyd C (2001) P34cdc2 kinase is associated with cortical microtubules from higher plant protoplasts. FEBS Lett 508:157-161

Hirano T (2000) Chromosome cohesion, condensation, and separation. Ann Rev Biochem 69:115-144

Hush JM, Wu L, John PCL, Hepler LH, Hepler PK (1996) Plant mitosis promoting factors disassembles the microtubule preprophase band and accelerates prophase progression in Tradescantia. Cell Biol Int 20:275-287

Jasencakova Z, Meister A, Schubert I (2001) Chromatin organization and its relation to replication and histone acetylation during the cell cycle in barley. Chromosoma 110:83-92

John PCL (1996) The plant cell cycle: conserved and unique features in mitotic control. Prog Cell Cycle Res 2:59-72
Kapraun DF (2005) Nuclear DNA content estimates in multicellular green, red and brown algae: phylogenetic considerations. Ann Bot 95:7-44

Kearsey SE, Labib K (1998) MCM proteins: evolution, properties, and role in DNA replication. Biochim Biophys Acta 1398:113136

King RW, Glotzer M, Kirschner MW (1996) Mutagenic analysis of the destruction signal of mitotic cyclins and structural characterization of ubiquitinated intermediates. Mol Biol Cell 7:13431357

Kohn EA, Ruth ND, Brown MK, Livingstone M, Eastman A (2002) Abrogation of the S phase DNA damage checkpoint results in S phase progression or premature mitosis depending on the concentration of 7-hydroxystaurosporine and the kinetics of Cdc25C activation. J Biol Chem 277:26553-26564

Kung AL, Sherwood SW, Schimke RT (1993) Differences in the regulation of protein synthesis, cyclin $\mathrm{B}$ accumulation, and cellular growth in response to the inhibition of DNA synthesis in Chinese hamster ovary and HeLa S3 cells. J Biol Chem 268:23072-23080

Kwiatkowska M, Maszewski J (1986) Changes in the occurrence and ultrastructure of plasmodesmata in antheridia of Chara vulgaris L. during different stages of spermatogenesis. Protoplasma 132:179-188

Lutzmann M, Maiorano D, Méchali M (2006) A Cdt1-geminin complex licenses chromatin for DNA replication and prevents rereplication during S phase in Xenopus. EMBO J 25:5764-5774

Maszewski J, van Bel AJE (1996) Intracellular transport of Lucifer Yellow in young and mature antheridia of Chara vulgaris L. Bot Acta 109:110-114

Merrill BJ, Holm C (1999) A requirement for recombinational repair in Saccharomyces cerevisiae is caused by DNA replication defects of mec1 mutants. Genetics 153:595-605

Mineyuki Y, Aioi H, Yamashita M, Nagahama Y (1996) A comparative study on stainability of preprophase bands by the PSTAIR antibody. J Plant Res 109:185-192

Navarrete MH, Cuadrado A, Cfinovas JL (1983) Partial elimination of G1 and G2 periods in higher plant cells by increasing the S period. Exp Cell Res 148:273-280

Nishihama R, Machida Y (2001) Expansion of the phragmoplast during plant cytokinesis: a MAPK pathway may MAP it out. Curr Opin Plant Biol 4:507-512

Osborn AJ, Elledge SJ, Zou L (2002) Checking on the fork: the DNAreplication stress-response pathway. Trends Cell Biol 12:509516

Pines J, Hunter T (1991) Human cyclins A and Bl are differentially located in the cell and undergo cell cycle-dependent nuclear transport. J Cell Biol 115:1-17

Polit JT, Maszewski J, Kaźmierczak A (2002) Effect of BAP and IAA on the expression of G1 and G2 control points and the G1-S and G2-M transitions in root meristem cells of Vicia faba. Cell Biol Int 27:559-566

Rao PN, Johnson RT (1970) Mammalian cell fusion: studies on the regulation of DNA synthesis and mitosis. Nature 225:159-164

Renaudin JP, Colasenti J, Rime H, Yuan Z, Sundaresan V (1994) Cloning of four cyclins from maize indicates that higher plants have three structurally distinct groups of mitotic cyclins. Proc Natl Acad Sci USA 91:7375-7379

Rodríguez-Bravo V, Guaita-Esteruelas S, Salvador N, Bachs O, Agell N (2007) Different S/M checkpoint responses of tumor and nontumor cell lines to DNA replication inhibition. Cancer Res 67:11648-11656

Rybaczek D, Maszewski J (2007a) DSB-dependent phosphorylation of H2AX histones and induction of PCC in hydroxyurea-treated root meristem cells of Raphanus sativus, Vicia faba and Allium porrum. Protoplasma 230:31-39 
Rybaczek D, Maszewski J (2007b) Induction of foci of phosphorylated $\mathrm{H} 2 \mathrm{AX}$ histones and premature chromosome condensation after DNA damage in root meristem cells of Vicia faba. Biol Plant 51:443-450

Sakano K, Oikawa S, Hasegawa K, Kawanishi S (2001) Hydroxyurea induces site-specific DNA damage via formation of hydrogen peroxide and nitric oxide. Jpn J Cancer Res 92:1166-1174

Samuels AL, Staehelin LA (1996) Caffeine inhibits cell plate formation by disrupting membrane reorganization just after the vesicle fusion step. Protoplasma 195:144-155
Sen R, Ghosh S (1998) Induction of premature mitosis in S-blocked onion cells. Cell Biol Int 22:867-874

Sogo JM, Lopes M, Foiani M (2002) Fork reversal and ssDNA accumulation at stalled replication forks owing to checkpoint defects. Science 297:599-602

Van Damme D (2009) Division plane determination during plant somatic cytokinesis. Curr Opin Plant Biol 12:745-751

Van't Hof J (1965) Relationships between mitotic cycle duration, S period duration and the average rate of DNA synthesis in the root meristem cells of several plants. Exp Cell Res 39:48-58 